fournal of Medical Genetics (1974). 11, 244.

\title{
Pleiotropic effects of the gene for retinoblastoma*
}

\author{
F. D. KITCHIN and ROBERT M. ELLSWORTH
}

\author{
From the College of Physicians and Surgeons and the Cancer Research Center, Columbia University, \\ 630 West 168th Street, New York, New York 10032, USA
}

\begin{abstract}
Summary. Vigorous treatment of retinoblastoma in the last 30 years has resulted in a large population of survivors with useful vision, in which the late effects of genetically associated tumours can be seen. An increase in second primary tumours, mainly osteogenic sarcoma, has been found in those children who carry the germinal mutations, and not in the majority of survivors of unilateral disease. The findings indicate a pleiotropic effect of the retinoblastoma gene which may act as an initiator in two forms of neoplasia.
\end{abstract}

Retinoblastoma is one of several rare tumours of childhood that has strong genetical associations. Segregation analysis of pedigrees in both familial retinoblastoma and in survivors of sporadic retinoblastoma has suggested that a germ cell mutation is a primary event when the disease is bilateral and that this mutation is expressed in the heterozygous state, with a manifestation of almost $100 \%$ (Ellsworth, 1969). In unilateral disease the genetical influences are not quite so straight-forward and it has been estimated that this heterogeneous group is composed of a small fraction (10-15\%) with germinal mutations of low manifestation and a larger group of phenocopies. In addition, there is a growing body of evidence to suggest that the locus for retinoblastoma is probably on the long arm of chromosome No. 13 (Taylor, 1970).

The introduction of radiotherapy in 1936, along with light coagulation and cryotherapy has so improved the outlook, that $80 \%$ now survive long term and retain useful vision. Thus many children with retinoblastoma now live to reproductive age and beyond and form a group in which the late biological effects of genetical tumours can be observed. The mortality that does occur is divided into two groups; that which is early, within 2 years of diagnosis and due to metastatic disease, and late death due to tumours at other sites either within or outside a radiation field. This report is concerned with the genetical analysis of second tumours in a group of treated retinoblastoma survivors.

Received 19 November 1973.

* Supported by the National Cancer Institute, grant No. CA 13696.

\section{Material and methods}

The population of retinoblastoma patients which form the basis of this study total 1130 . The vast majority were seen and treated at the Institute of Ophthalmology of the Columbia Presbyterian Medical Center in New York by Dr A. B. Reese and Dr R. M. Ellsworth.

For illustrative purposes we have chosen the years 1950-63, inclusive, to demonstrate any major differences between the unilateral and bilateral groups. For during these years there was a reasonable uniformity of treat-? ment and 10 years provides an adequate follow up period. In the majority of epidemiological and demographic studies of retinoblastoma, $25-30 \%$ of new cases are bilateral, but in the present series due to a referral bias the figure is $66 \%$.

\section{Results}

Table I demonstrates that $15 \%$ of unilateral cases (uni) die of metastatic disease compared with $11 \%$ of bilateral cases (bi). This difference is not significant $\left(\chi_{1}^{2}=0 \cdot 6 ; p>0 \cdot 4\right)$. The $7 \%$ that develop a second tumour either within or outside a radiation field do so after a mean latent period of 13 years. The numbers also demonstrate the remarkable increase in tumours in the bilaterally affected and the absence of extra-ocular tumours in adult survivors of unilateral retinoblastoma. Since unilateral retinoblastoma is usually treated by enucleation and not radiotherapy, no radiation induced tumours are anticipated in this group.

Because of these findings we have ascertained as fully as possible all examples of second tumours occurring in our population of retinoblastoma survivors. Some of these have been previously re- 
TABLE I

\begin{tabular}{c|c|c|c|c|c|c}
\hline $\begin{array}{c}\text { Year } \\
\text { Treated }\end{array}$ & \multicolumn{2}{|c|}{ No. Seen } & \multicolumn{2}{|c|}{ Metastatic Disease } & \multicolumn{2}{|c}{ Second Tumour } \\
\cline { 2 - 4 } & Uni & $\mathrm{Bi}$ & $\mathrm{Uni}$ & $\mathrm{Bi}$ & $\mathrm{Uni}$ & $\mathrm{Bi}$ \\
\hline $1950-1963$ & 179 & 326 & $27(15 \cdot 1 \%)$ & $46(11 \cdot 1 \%)$ & 0 & $24(7 \cdot 3 \%)$ \\
\hline
\end{tabular}

TABLE II

SECOND TUMOURS IN ALL BILATERAL RETINOBLASTOMA SURVIVORS

\begin{tabular}{l|c|c|c}
\hline \multicolumn{1}{|c|}{ Type } & $\begin{array}{c}\text { Within } \\
\text { Radiation } \\
\text { Field }\end{array}$ & Outside Field & Total \\
\hline $\begin{array}{l}\text { Sarcoma } \\
\text { Soft tissue } \\
\text { Bone }\end{array}$ & $\begin{array}{l}17 \\
16\end{array}$ & 0 & 40 \\
\hline Squamous epithelioma & 2 & 2 & 2 \\
\hline Thyroid & 35 & 1 & 2 \\
\hline Wilms & 10 & 45 \\
\hline Total & & 2 & 1 \\
\hline
\end{tabular}

* Includes one unilateral retinoblastoma.

ported (Sagerman et al, 1969; Jensen and Miller, 1971). They have been graded as to site, type, and whether the retinoblastoma was unilateral or bilateral.

Table II illustrates the findings. An impressive number of survivors have developed a second tumour, about $4 \%$ of the total series, and with one exception confined to children with bilateral disease. Most outstanding is the occurrence of seven examples of osteogenic sarcoma-three previously reported (Jensen and Miller, 1971)-in long bones, far removed from the radiation field.

If we assume an average yearly incidence of osteogenic sarcoma as 1 in 100,000 (Doll et al, 1970), a 40 -year risk period in a population of 800 would provide 0.32 cases. The seven observed cases represent a highly significant excess when referred to the Poisson distribution.

In unilateral cases about $10-15 \%$ are the result of germinal mutations expressed only in one eye, and so the expected number of unilateral survivors in our population with second tumours is $3-4$; one was found. This child developed thyroid carcinoma following radiation therapy to the orbit after enucleation. The remaining bilaterally affected children (44) had all received radiation therapy to the remaining eye after enucleation of the most advanced eye, with one exception. He developed a sarcoma in the temporal fossa that would have been considered from its cranial site to be radiation induced but for the fact he received no radiotherapy and was treated by bilateral enucleation

\section{Discussion}

Since genetical analysis has shown that the first step in bilateral disease is a germinal mutation, it follows that all cells must carry the abnormal gene. Our results demonstrate the striking increase in second primary tumours in survivors of bilateral retinoblastoma who carry the mutation and not in the majority of survivors of unilateral disease. Since clearly it is a mutated portion of the genome, not cancer that is inherited, it appears that the germinal mutation is but the first in a multistep process of neoplastic transformation.

We can postulate that in retinoblastoma this germinal mutation acts as an initiator by increasing the liability of all the retinal cells to undergo neoplastic transformation after a second mutagenic episode. Knudson (1971) has analysed this hypothesis mathematically and arrived at some rough estimate of the frequency of the second mutation. In addition, the second step is temporally restricted for in our series new tumours do not develop after the age of 33 months. This suggests that the period at risk is limited and probably related to the fact that at some time as yet undetermined in man, the retinal neuroblasts become post mitotic and differentiation ensues. In other vertebrates (Sidman, 1961; Fujita and Horii, 1963; Jacobson, 1968;-Hollyfield, 1972), differentiation proceeds from the central to the peripheral retina, and if similar events take place in the human eye, it appears that the population of cells still undergoing mitosis and possibly at risk becomes gradually reduced and situated anterior to the equator.

From our present results it is difficult to avoid the conclusion that the retinoblastoma locus is also concerned with the initiation of other tumoursparticularly osteogenic sarcoma of the long bones. This pleiotropic effect may not be restricted to bone tumours at distant sites but may be hidden in the subgroup which we have characterized as radiation induced. For it is not possible to assess any increase in liability to post-irradiation tumours in the bilateral survivors as no control group with comparable radiotherapy and follow up is presently available.

At a molecular level it is difficult to imagine how the retinoblastoma locus sensitizes both retinoblasts and osteoblasts, unless some fundamental step in DNA biochemistry is involved. If this is so, it could be concerned with DNA polymerase enzyme systems, either DNA or RNA dependent, that 
control the fidelity of DNA synthesis or DNA repair enzymes which correct faults in duplication. Such mutator genes have been described in $E$. coli and also in man (Epstein, Fukuyama, and Epstein, 1971), and it should be possible to examine some of these systems in several tissues of retinoblastoma patients with the hereditary form of this disease.

\section{Addendum}

Since this paper was submitted three more examples of second primary tumours of the femur have been observed in the bilateral retinoblastoma survivors; two cases of osteosariomas and one case of Ewing's sarcoma.

\section{RBFERENCES}

Doll, R., Muir, C., and Waterhouse, J. (eds). (1970). Cancer Incidence in Five Continents. Springer, Berlin, Heidelberg, New York.

Ellsworth, R. M. (1969). The practical management of retinoblastoma. Transactions of the American Ophthalmological Society 67, 462-534.
Epstein, W. L., Fukuyama, K., and Epstein, J. H. (1971). Ultraviolet light, DNA repair and skin carcinogenesis in man. Federation Proceedings, 30, 1766-1771.

Fujita, S. and Horii, M. (1963). Analysis of cytogenesis in chick retina and tritiated thymidine autoradiography. Archivum Histologicum Faponicum, 23, 359-366.

Hollyfield, J. G. (1972). Histogenesis of the retina in the killifish, fundulus heteroclitus. Fournal of Comparative Neurology, 144, 373-380.

Jacobson, M. (1968). Cessation of DNA synthesis in retinal ganglion cells correlated with the time of specification of their central connections. Developmental Biology, 17, 219-232.

Jensen, R. D. and Miller, R. W. (1971). Retinoblastoma: epidemiologic characteristics. New England fournal of Medicine, 285, 307-311.

Knudson, A. G., Jr. (1971). Mutation and cancer: statistical study of retinoblastoma. Proceedings of the National Academy of Sciences, 68, 820-823.

Sagerman, R., Cassady, J. R., Tretter, P., and Ellsworth, R. M. (1969). Radiation induced neoplasia following external beam therapy for children with retinoblastoma. American fournal of Roentgenology, 105, 529-535.

Sidman, R. L. (1961). Histogenesis of mouse retina studied with thymidine-3H. In Structure of the Eye, ed. by G. K. Smelser, pp. 487-506. Academic Press, New York.

Taylor, A. I. (1970). $\mathrm{Dq}^{-}$, Dr and retinoblastoma. Humangenetik, 10, 209-217. 